\title{
An unusual presentation of secondary pleural hydatidosis
}

\author{
X. Aguilar*, J. Fernández-Muixí**, R. Magarolas*, A. Saurít, F. Vidal**, C. Richart**
}

An unusual presentation of secondary pleural hydatidosis. X. Aguilar, J. FernándezMuixí, R. Magarolas, A. Saurí, F. Vidal, C. Richart. (CERS Journals Ltd 1998.

ABSTRACT: Although pleural involvement is relatively common in cystic hydatid disease, one of the rarest and least known complications is secondary pleural hydatidosis.

We report the case of a patient who presented with polycystic secondary pleural hydatidosis 4 yrs after treatment for a pyopneumothorax caused by rupture of a pulmonary cyst near the pleural space. Due to the coexistence of severe chronic obstructive pulmonary disease, surgery was ruled out.

The patient was treated with albendazole, with a favourable outcome. Eur Respir J 1998; 11: 243-245.

Cystic hydatid disease (CHD) is an infection produced by larvae of the parasite platyhelminth Echinococcus granulosus [1]. It has a wide geographic distribution and humans may be infected incidentally as intermediate hosts in the parasite's life cycle. The organs most commonly affected are the liver and the lungs [1, 2]. Pleural involvement is rare, and usually follows the rupture of a pulmonary or hepatic cyst inside the pleural space [3]. Since in about $90 \%$ of episodes the cyst is no longer fertile after rupture, secondary pleural hydatidosis (SPH) is a rare event occurring in less than $10 \%$ of such cases [3]. Surgery is the treatment of choice for CHD, but antihelmintic therapy has been advocated in patients with recurrent hydatidosis or in those in whom surgical intervention involves a high risk of morbidity or mortality [4]. This report describes an unusual case of a SPH treated successfully with albendazole.

a)

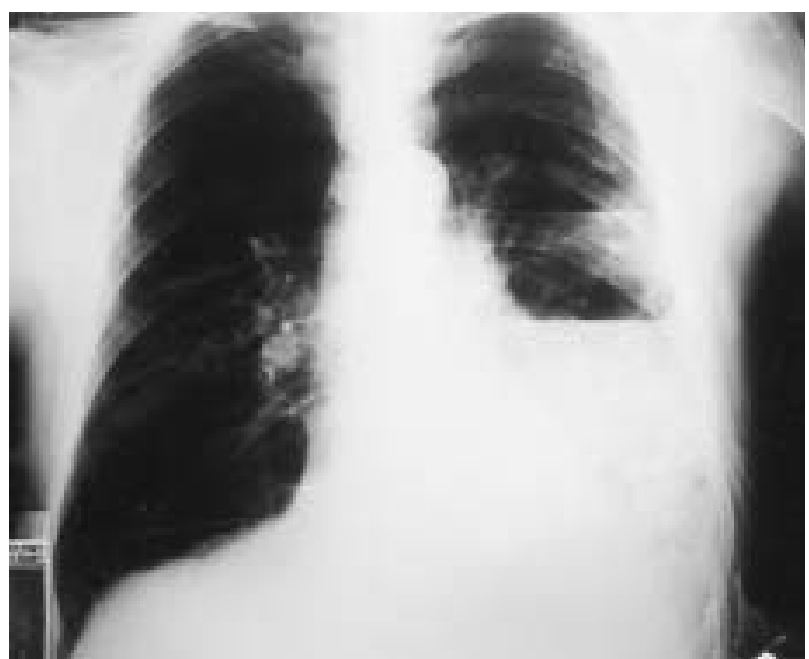

*Respiratory Unit, **Internal Medicine Service and +Radiology Service, Joan XXIII University Hospital, Faculty of Medicine and Health Sciences, University Rovira i Virgili, Tarragona (Catalonia), Spain.

Correspondence: X. Aguilar, Unitat de Pneumologia (6a. planta), Hospital Universitari Joan XXIII, C/ Dr. Mallfrè Guasch 4, (43007) Tarragona (Catalonia), Spain. Fax: 0034977224011

Keywords: Albendazole, cystic hydatid disease, secondary pleural hydatidosis

Received: August 141996

Accepted after revision June 251997

\section{Case report}

A 66 yr old male resident of Tarragona (Catalonia, Spain) was admitted to hospital with left pleuritic pain and increasing dyspnoea of 2 months duration. He was a tobacco smoker (45 pack-years). His past history included a surgical excision of a hepatic hydatid cyst at the age of $40 \mathrm{yrs}$, and the diagnosis of severe chronic obstructive pulmonary disease (COPD) (forced expiratory volume in one second (FEV1) $1.05 \mathrm{~L}, 35 \%$ of predicted value) with bullous emphysema at $50 \mathrm{yrs}$ of age. He was receiving treatment with bronchodilator drugs.

Four years prior to the current admission, he had had left pyopneumothorax (fig. 1a), which was diagnosed as a complication of the rupture of an infected emphysematous bulla and was successfully treated with closed thoracic drainage and antimicrobial drugs. Physical

b)

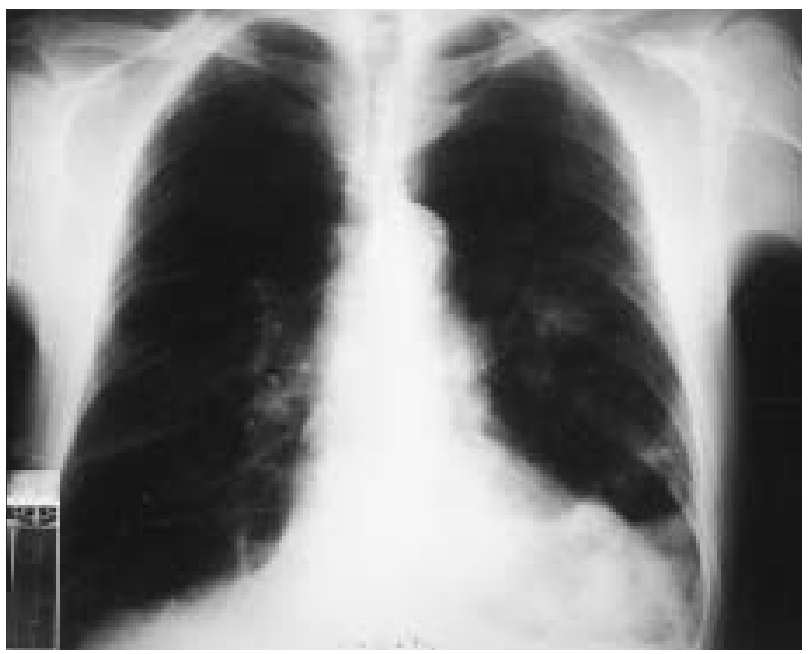

Fig. 1. - a) Posteroanterior chest radiograph at the first admission (4 yrs prior to the current admittance) shows left pyopneumothorax. b) Chest radiograph on actual admission indicates multiple nodular images in the left lower hemithorax and diffuse bullous emphysema. 
examination was normal except for bilateral hypophonesis on chest auscultation. Chest radiographs were consistent with bullous emphysema and there were diverse nodular images in the left lower hemithorax (fig. 1b).

Main biochemical and electrocardiographic (ECG) data were normal. Arterial blood gas values, on room air, revealed mild hypoxaemia (arterial oxygen tension $\left(\mathrm{Pa}, \mathrm{O}_{2}\right) 10 \mathrm{kPa}(76 \mathrm{mmHg})$. Blood eosinophil count was 514 eosinophils $\mathrm{mm}^{-3}$ (table 1 ). Total immunoglobulin $\mathrm{E}(\mathrm{IgE})$ was $2,850 \mathrm{U} \cdot \mathrm{mL}^{-1}$ with a positive radioallergosorbent test (RAST) specific IgE for E. granulosus (2.10 Phadebas RAST units $\left.(\mathrm{PRU}) \cdot \mathrm{mL}^{-1}\right)$ (table 1$)$.

Table 1. - Total IgE, specific RAST IgE (Echinococcus granulosus) and eosinophilia on admission and after therapy

\begin{tabular}{lccc}
\hline & $\begin{array}{c}\text { On } \\
\text { admission }\end{array}$ & $\begin{array}{c}\text { After } \\
\text { therapy } \\
\text { 3rd cycle }\end{array}$ & $\begin{array}{c}\text { After } \\
\text { therapy } \\
\text { 6th cycle }\end{array}$ \\
\hline Eosinophils $\mathrm{n} \cdot \mathrm{mm}^{-3}$ & 514 & 215 & 80 \\
Total IgE $\mathrm{U} \cdot \mathrm{mL}^{-1}$ & 2,850 & 911 & 825 \\
RAST IgE PRU $\cdot \mathrm{mL}^{-1}$ & 2.10 & 1.31 & 1.07
\end{tabular}

IgE: immunoglobulin E; RAST: radioallergosorbent test; PRU: Phadebas RAST units.
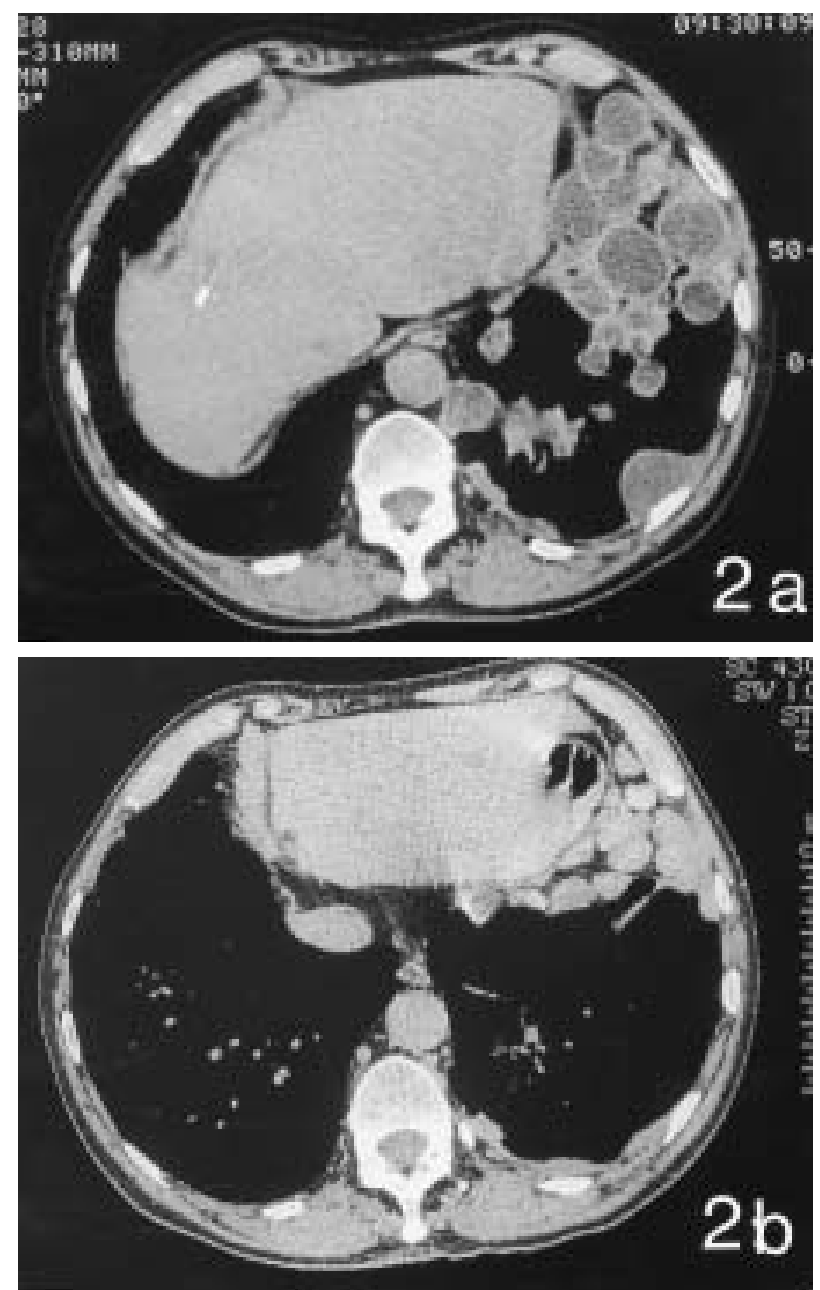

Fig. 2. - a) Chest computed tomography (CT) scan indicates multiple cysts located in the left lower hemithorax and implanted in both diaphragmatic and mediastinic pleura. b) Chest CT scan (after therapy with albendazole) shows a reduction in the number and size of the cysts, which became completely opacified.
Determinations of antinuclear antibodies, anti-deoxyribonucleic acid (DNA), rheumatoid factor, carcinoembryonic antigen (CEA) and alpha ${ }_{1}$-antitrypsin were either normal or negative. The tuberculin skin test with two tuberculin units (TU) purified protein derivative (PPD), RT-23, was negative. Examination of three samples of sputum showed no acid-fast bacilli, fungi or atypical cells

Thoracic computed tomographic (CT) scan revealed multiple bullae diffusely distributed in both lungs, and cysts located in the anterior lower left hemithorax. These cysts were implanted both in the diaphragmatic and mediastinic pleura (fig. 2a). A residual calcified lesion in the right liver lobe was also noted.

SPH was diagnosed and, as surgery was contraindicated, treatment was initiated with albendazole, 10 $\mathrm{mg} \cdot \mathrm{kg}^{-1}$ daily, in cycles of 28 days followed by 14 days with no treatment. Clinical status as well as analytical data of the patient improved progressively, as reflected in table 1. After the sixth cycle, thoracic CT scan showed a marked reduction in the number and size of the cysts, which subsequently became completely opacified (fig. 2b).

\section{Discussion}

Human echinococcosis is caused by three species of Echinococcus: E. granulosus, E. Multilocularis and E. vogeli $[1,2]$. E. granulosus produces $\mathrm{CHD}$, which is the commonest form in humans, representing some $90 \%$ of cases [5]. CHD is found worldwide. Spain is an endemic zone, and its annual incidence is estimated at 5.21-7.55 cases per 100,000 inhabitants [6]. CHD of the lung is asymptomatic in $30 \%$ of patients [4], and, when symptoms occur, they are usually due to the compression of the underlying pulmonary tissue by the cyst and/or to the presence of complications, such as rupture or infection $[2,5]$. A hydatid cyst may be confused with a bulla if it cracks and becomes empty [7]. This may have been the case in our patient when he presented pyopneumothorax 4 yrs before developing SPH.

Although, in rare instances, SPH has been associated with a haematogenous dissemination of the larvae, usually it is caused by a rupture of a neighbouring cyst with dissemination of the contents of the cyst (multiple daughter cysts and scolices) along the pleura. Less than $10 \%$ of these patients develop SPH [3]. This low percentage has been attributed to bacterial superinfection of the pleural space [3]. Three different forms of SPH have been described: pleural granulomatosis, hydatidothorax, and pleural hydatid graft [3]. The case reported here is a peculiar example of this last form. In endemic zones, hydatic cysts are among the major causes of well-defined nodular pulmonary lesions [5].

Medical therapy is useful when surgery is technically difficult or contraindicated [1-5]. Albendazole is the drug of choice, and the usual dose is $10-15 \mathrm{mg} \cdot \mathrm{kg}^{-1}$ daily for 4 weeks in repeated cycles (usually more than three), separated by 2 weeks without treatment $[1$, 8].

This case is an example of secondary pleural hydatidosis which emphasizes that when pleural effusion and/ 
or pyopneumothorax are detected in a patient with previous hepatic cystic hydatid disease, the possibility of secondary pleural dissemination should be considered.

\section{References}

1. King CH. Cestodes (tapeworms). In: Mandell GL, Bennett JE, Dolin R, eds. Principles and Practice of Infectious Diseases. 4th Edn. New York, Churchill Livingstone Inc., 1995; pp. 2544-2552.

2. Case records of the Massachusetts General Hospital. Case 45-1987. N Engl J Med 1987; 317: 1209-1218.

3. Skerrett SJ, Plorde JJ. Parasitic infections of the pleural space. Semin Respir Med 1992; 13: 242-258.
4. Dogan R, Yürsel M, Cetin G, et al. Surgical treatment of hydatid cysts of the lung: report on 1,055 patients. Thorax 1989; 44: 192-199.

5. Kennedy D, Sharma OP. An unusual presentation of hydatid disease of the lungs. Chest 1990; 97: $997-$ 999.

6. Caylà JA, Barquet N, Muñoz C, et al. Estudio epidemiológico de la hidatidosis humana en Catalunya (1977-1981) (I).Med Clin (Barc) 1986; 86: 397-404.

7. Gouliamos AD, Kalovidouris A, Papailiou J, Vlahos L, Papavasiliou C. CT appearance of pulmonary hydatid disease. Chest 1991; 100: 1578-1581.

8. Aggarwai P, Wali JP. Albendazole in the treatment of pulmonary echinococcosis. Thorax 1991; 46: 599-600. 